\title{
Thin film solar cells on glass by transfer of monocrystalline Si films
}

\author{
R. B. Bergmann, ${ }^{\dagger}$ T. J. Rinke, R. M. Hausner, M. Grauvogl, \\ M. Vetter, and J. H. Werner \\ Institute of Physical Electronics, University of Stuttgart, Pfaffenwaldring 47, \\ D-70569 Stuttgart, Germany
}

\begin{abstract}
Thin film solar cells based on monocrystalline Si films are transferred to a glass superstrate. Chemical vapor deposition serves to epitaxially deposit Si on quasi-monocrystalline Si films obtained from thermal crystallization of a double layer porous Si film on a Si wafer. A separation layer that forms during this crystallization process allows one to separate the epitaxial layer on top of the quasi-monocrystalline film from the starting Si wafer. We presently achieve an independently confirmed solar cell conversion efficiency of $9.26 \%$. Ray tracing studies in combination with electrical device simulation indicate an efficiency potential of around $17 \%$ using simple device processing and moderate assumptions on minority carrier lifetime and surface recombination.
\end{abstract}

\section{INTRODUCTION}

Solar cells fabricated from monocrystalline Si wafers have achieved a conversion efficiency of 24.4\% [1]. Wafer thinning to a thickness of around $50 \mu \mathrm{m}$ still allows one to realize a cell efficiency of $21.5 \%$ [2]. However, cost issues have up to now, in contrast to applications in the area of microelectronics [3], inhibited the use of thin monocrystalline Si films in photovoltaics. Therefore, since a few years, a number of processes are in the stage of development, that employ the transfer of monocrystalline Si films onto a foreign substrate [4]. The most prominent approaches currently are

(i) the formation of a net-like monocrystalline $\mathrm{Si}$ structure by the so called Epi-lift-technique [5],

(ii) the formation of thin, monocrystalline Si-waffles using the so-called $\Psi$-process [6],

(iii) epitaxial growth of monocrystalline Si films on crystallized porous Si introduced by Sony Corp. [7], and

(iv) the formation of so-called quasi-monocrystalline Si (QMS) films with internal light trapping introduced at our institute $[8,9]$. Apart from the approach of Sony Corp., no solar cell results have been reported so far.

In this paper, we describe a solar cell technology based on the transfer of monocrystalline Si films onto a glass superstrate. Independent from the work of Sony Corp., we developed a process that realizes the formation of monocrystalline Si films by thermal annealing of porous Si. The crystallization of a several $\mu \mathrm{m}$ thick film of low porosity on a thin film with higher porosity, forms a quasi-monocrystalline Si (QMS) film on a separation layer, that enables the transfer of the QMS Si film from a reusable Si substrate to glass. In contrast to poly- and microcrystalline Si based thin film solar cells, this approach makes full benefit of the efficiency potential of monocrystalline Si using comparatively simple device manufacturing technology.

† ralf.bergmann@ipe.uni-stuttgart.de

\section{RESULTS AND DISCUSSION}

The first part of the paper describes the formation of the quasi-monocrystalline Si film, the second part gives details on the solar cell device process, while the third part outlines results of device simulations using a combination of ray tracing and one dimensional electrical device simulation.

2.1. Formation of quasi-monocrystalline Si films. Device fabrication is based on the formation of a doublelayer porous Si film that is crystallized at elevated temperatures [8]. Figure 1a shows a cross section of a crystallized Si film and a separation layer between the QMS film and the Si wafer. The morphology of the Si film strongly depends on film thickness. Films with a thickness exceeding one $\mu \mathrm{m}$ contain a high density of voids, as is clearly visible in the cross section shown in Figure $1 \mathrm{~b}$. Due to the presence of voids, films are termed quasi-monocrystalline. As schematically shown in Figure $1 \mathrm{c}$, these voids lead to diffuse light scattering and, depending on film thickness, to light trapping within the crystalline film. Up to 30 porous Si films have been produced from one Si wafer up to now, demonstrating the reusability of the Si starting wafer. For further details of the film formation process see [10].

2.2. Solar cell fabrication and transfer to glass. Solar cell fabrication involves

(i) epitaxial growth of the monocrystalline Si absorber film on top of the QMS film,

(ii) $p n$-junction and contact grid formation,

(iii) anti-reflection coating,

(iv) transfer to a glass superstrate and

(v) formation of a back contact.

Figure 2 schematically outlines the cell formation process.

After crystallization of the porous Si film, see Figure 2a, high temperature chemical vapor deposition 
(a)

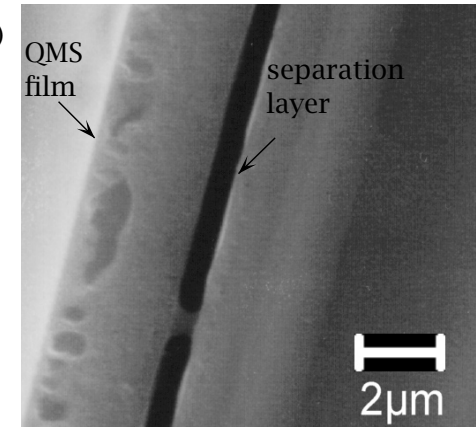

(b)
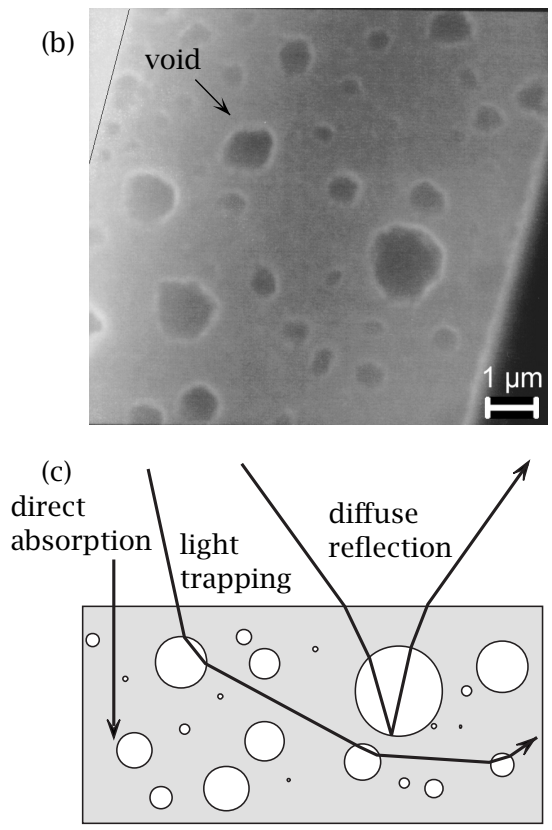

(a)

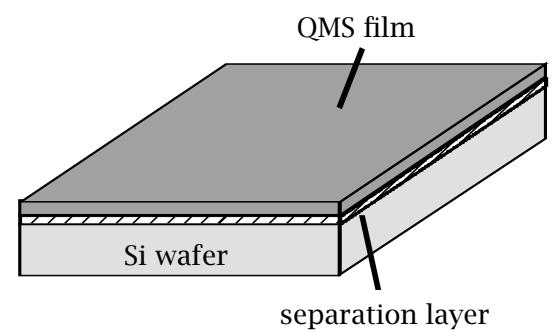

(b)

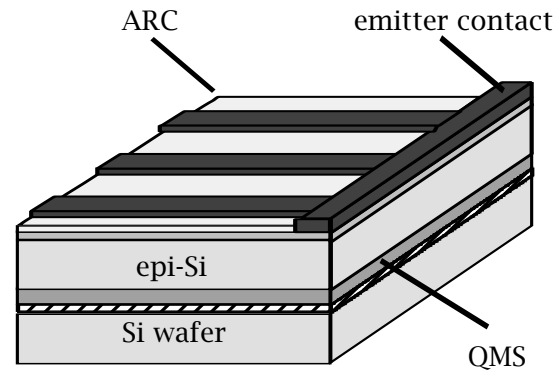

(c)

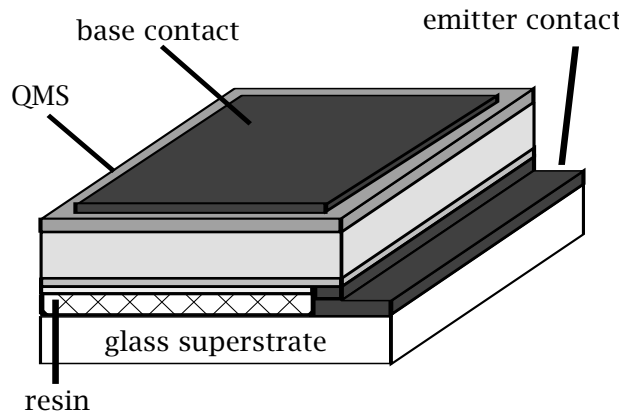

Figure 1. Morphology and interaction with light in quasimonocrystalline Si (QMS) films: a) Cross section of a crystallized Si film and a separation layer between the QMS film and the Si wafer. b) Cross section of an $8 \mu \mathrm{m}$ thick QMS film that contains a high density of voids, scanning electron micrographs. c) Schematic cross section of a QMS film: Apart from direct absorption of sunlight, the presence of voids leads, depending on film thickness, to diffuse light scattering and light trapping within the crystalline film.

from $\mathrm{SiHCl}_{3}$ at a temperature of $1100{ }^{\circ} \mathrm{C}$ forms an epitaxial layer with a thickness between 12 and $50 \mu \mathrm{m}$ on top of the QMS film, see Figure $2 \mathrm{~b}$. In the present device configuration, we first deposit a Si film with a thickness of a few $\mu \mathrm{m}$ and a doping concentration of $3 \times 10^{19} \mathrm{~cm}^{-3}$ that acts as a back surface field followed by an absorber film with a doping concentration of $3 \times 10^{17} \mathrm{~cm}^{-3}$. The QMS film does therefore not contribute to photovoltaic current generation.

Formation of an emitter is accomplished by phosphorus diffusion at $830^{\circ} \mathrm{C}$ for $40 \mathrm{~min}$ to obtain an emitter with a sheet resistance of $40 \Omega / \square$ after the final preparation of a $30 \mathrm{~nm}$ thick oxide via thermal oxidation at $1000{ }^{\circ} \mathrm{C}$ for $12 \mathrm{~min}$. Photolithography serves to form a front contact grid that consists of $\mathrm{Ti} / \mathrm{Pd} / \mathrm{Ag}$. A $30 \mathrm{~nm}$
Figure 2. Solar cell processing. a) QMS film on a Si wafer. b) Epitaxial absorber film on top of the QMS film, emitter formation via phosphorus diffusion, photolithography forms front contact grid and $\mathrm{SiN}_{x}$ film serves as an anti-reflection coating (ARC). c) Semi-finished device is attached to glass superstrate by use of an organic resin and then separated from starting wafer. Evaporation of $\mathrm{Pd} / \mathrm{Al}$-contact finalizes the device formation.

thick $\mathrm{SiN}_{x}$ film deposited at room temperature by reactive rf-magnetron sputtering functions as an antireflection coating between the Si solar cell and the glass superstrate. These non-stochiometric sputtered films have a small Si surplus that results in a refractive index of 2.0 to 2.1 at $633 \mathrm{~nm}$. The semi-finished device is attached to glass by use of an organic resin. The action of mechanical force allows one to separate the film from the starting wafer. An evaporated $\mathrm{Pd} / \mathrm{Al}$-contact finalizes the device structure that is schematically depicted in Figure 2c.

Figure 3 depicts a photograph of a $12 \mu \mathrm{m}$ thick epitaxial Si film deposited on a $2 \mu$ m thick QMS film that is detached from the starting $4^{\prime \prime}$-sized Si wafer and attached to a $3^{\prime \prime}$-sized glass substrate. 


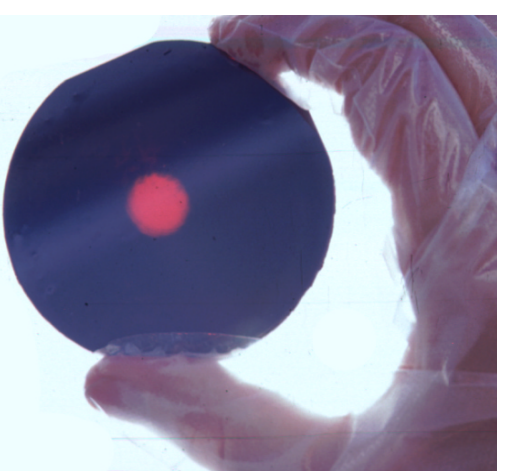

Figure 3. Photograph of a $12 \mu \mathrm{m}$ thick epitaxial Si film deposited on a $2 \mu \mathrm{m}$ thick OMS film attached to a $3^{\prime \prime}$-sized glass substrate. The film is held against the sun which is visible with some of it's red spectrum in the center of the film.

Figure 4 shows the illuminated current-voltage characteristics of a thin film solar cell on glass measured at the calibration laboratory of the Fraunhofer Institute for Solar Energy Systems in Freiburg, Germany. We presently achieve a confirmed cell efficiency of $9.26 \%$ under AM 1.5 G illumination for a solar cell with an area of $1.001 \mathrm{~cm}^{2}$. The thickness of the epitaxial Si film is $49 \mu \mathrm{m}$ including a $3 \mu \mathrm{m}$ thick back surface field deposited onto the $2 \mu \mathrm{m}$ thick QMS film. Internal quantum efficiency measurements reveal an effective diffusion length of $14.4 \mu \mathrm{m}$. Presently, a high dislocation and stacking fault density of several $10^{5} \mathrm{~cm}^{-2}$ limits the diffusion length in the epitaxial film. Reduction of defect density and thus a boost of the minority carrier diffusion length is expected to result in a significant increase in cell efficiency.

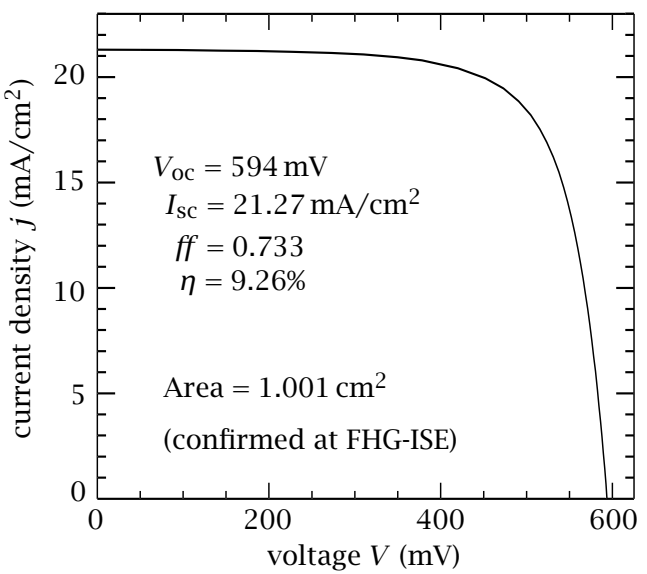

Figure 4. Illuminated current-voltage characteristic of a thin film solar cell on glass. Cell efficiency is $9.26 \%$ under AM $1.5 \mathrm{G}$ illumination confirmed at the Fraunhofer Institute for Solar Energy Systems in Freiburg, Germany. The cell has a $49 \mu \mathrm{m}$ thick epitaxial Si film and a cell area of $1.001 \mathrm{~cm}^{2}$.
2.3. Optical and electrical modeling of thin film solar cells. In order to determine the optical absorption and thus the maximum current density $j_{\text {scmax }}$ achievable using a thin monocrystalline Si film on a glass superstrate, we simulate a device structure schematically depicted in Figure 5a. The front surface is coated with a $70 \mathrm{~nm}$ thick $\mathrm{Si}_{3} \mathrm{~N}_{4}$ film, the rear surface consists of an $\mathrm{Al}$-contact and the Si absorber film thickness is in the range of 5 to $50 \mu \mathrm{m}$. Optical device simulation uses the Monte-Carlo ray tracing program SUNRAYS [11]. Three basic cases are distinguished in this simulation:

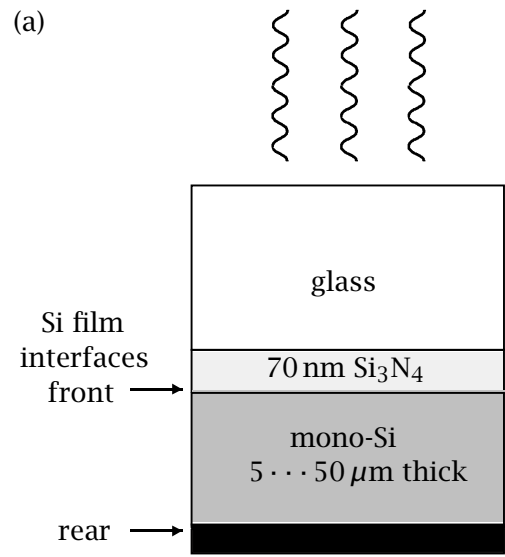

rear $\mathrm{Al}$ contact

(b)

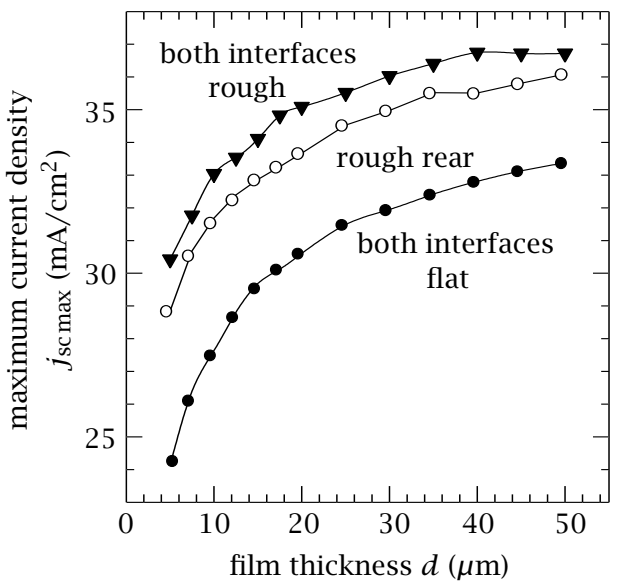

Figure 5. a) Schematic cross section of a Si thin film structure on glass used for optical simulation. A $\mathrm{Si}_{3} \mathrm{~N}_{4}$ film serves as anti-reflection coating between the glass superstrate and the Si film, an Al film contacts the rear cell surface. b) Maximum current density $j_{\mathrm{scmax}}$ as calculated from SUNRAYS using the configuration shown in (a) depends on film thick ness $d$ and optical confinement: $i)$ Both $\mathrm{Si}$ interfaces are flat, ii) the rear $\mathrm{Si}$ interface is optically rough, and iii) both $\mathrm{Si}$ interfaces are optically rough.

(i) both Si interfaces are flat,

(ii) the rear Si interface is rough and of Lambertian character, and

(iii) both Si interfaces are rough and of Lambertian character. 
The simulation does not make any assumptions on the specific realization of the surface roughness. Figure $5 \mathrm{~b}$ shows the increase of $j_{\text {scmax }}$ with Si film thickness in the range of 5 to $50 \mu \mathrm{m}$. A substantial increase in $j_{\text {scmax }}$ is already gained from the presence of one optically rough surface, while the presence of a second optically rough surface is only of minor importance.

As indicated in Figure 1c, a QMS film may serve as a source of diffuse reflection of light. We therefore expect the current density $j_{\text {scmax }}$ generated in an epitaxial Si film on a QMS film to be between the current densities given for two flat surfaces and a flat front surface in combination with a rough rear surface. Such current densities can, of course, only be extracted from an idealized solar cell with a quantum efficiency being unity. We therefore use an electrical device simulator to model the influence of recombination parameters in the solar cell.

Modeling of electrical solar cell performance requires realistic assumptions on the cell structure and on the recombination parameters. As we aim at a simple and easily manufacturable device structure, the following assumptions are made with respect to recombination parameters: front surface recombination velocity is set to $S_{\mathrm{f}}=1000 \mathrm{~cm} / \mathrm{s}$ and back surface recombination velocity to $S_{\mathrm{b}}=10^{4} \mathrm{~cm} / \mathrm{s}$. Doping of the Si film is p-type with a concentration of $3 \times 10^{17} \mathrm{~cm}^{-3}$. We assume the quasi-monocrystalline $\mathrm{Si}$ film to act as a back surface field (BSF) with a uniform doping of $10^{19} \mathrm{~cm}^{-3}$ and a thickness of $1 \mu \mathrm{m}$. Optical generation profiles calculated by SUNRAYS are used for the device simulator PC-1D [12]. Both Si film thickness $d$ and minority carrier diffusion length $L$ are varied in the range of 5 to $50 \mu \mathrm{m}$.

Figure 6 a schematically shows the device structure described above, while Figure $6 \mathrm{~b}$ summarizes the results of the electrical device simulation. The conversion efficiency $\eta$ depends both on diffusion length $L$ and on device thickness $d$, all other parameters are kept constant. The plots in Figure $6 \mathrm{~b}$ show the maximum conversion efficiency $\eta_{\max }$ as a function of the minority carrier diffusion length $L$ for an optimum film thickness $d$ at each diffusion length. According to our modeling, a conversion efficiency of $\eta=17 \%$ requires a diffusion length of $L \approx 40 \mu \mathrm{m}$ and a film thickness $d \approx 25 \mu \mathrm{m}$ assuming an optically rough rear Si interface.

\section{CONCLUSIONS}

The transfer of monocrystalline Si films to a glass superstrate presents an innovative approach to realize efficient thin film solar cells. While first cell results demonstrate a cell efficiency of $9.26 \%$, further optimization of material quality and cell processing are expected to result in cell efficiencies comparable to conventional wafer based Si solar cells. Optical and electrical device modeling indicates, that a conversion efficiency in the range of $17 \%$ appears attainable with this process.
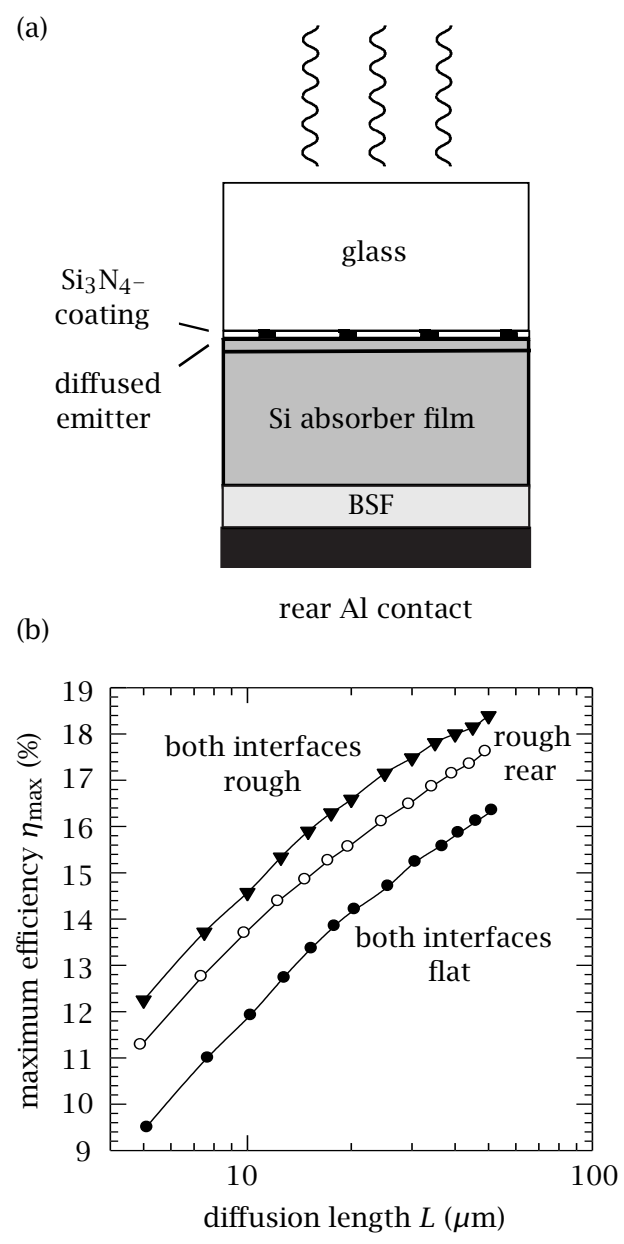

Figure 6. a) Schematic cross section of device structure for simulation sing PC-1D. Recombination velocity at front surface is set to $S_{\mathrm{f}}=1000 \mathrm{~cm} / \mathrm{s}$, surface recombination at rear side is $S_{\mathrm{b}}=10^{4} \mathrm{~cm} / \mathrm{s}$, Si film thickness is in the range of 5 to $50 \mu \mathrm{m}$. b) Maximum efficiency $\eta_{\max }$ for optimized film thickness as function of diffusion length $L$ (further explanation see text).

\section{ACKNOWLEDGEMENTS}

The authors wish to thank J. Schirmer and B. Winter for indispensable technical assistance, W. Appel from the Institute for Microelectronics in Stuttgart for chemical vapor deposition and C. Koch for carefully reading the manuscript. This work was supported by the European Commission within the CRYSTAL project under contract no. JOR3-CT97-0126 and the German Ministry for Education, Science, Research and Technology (BMBF) under contract no. 0329818.

\section{REFERENCES}

[1] J. Zhao, A. Wang, M. A. Green, and F. Ferrazza, Appl. Phys. Lett. 73 (1998), 1991.

[2] A. Wang, J. Zhao, S. R. Wenham, and M. A. Green, Prog. Photovolt. 4 (1996), 55.

[3] M. Bruel, B. Aspar, and A.-J. Auberton-Herve, Jpn. J. Appl. Phys. 36 (1997), 1636.

[4] R. B. Bergmann, Appl. Phys. A 69 (1999), 187. 
[5] K. J. Weber, K. Catchpole, M. Stocks, and A. W. Blakers, Proc. 26th IEEE Photovoltaic Specialists Conf., IEEE, Piscataway, 1997, p. 107.

[6] R. Brendel, H. Artmann, S. Oelting, W. Frey, J. H. Werner, and J. H. Queisser, Appl. Phys. A 67 (1998), 151.

[7] H. Tayanaka, K. Yamauchi, and T. Matsushita, Proc. 2nd World Conference on Photovoltaic Solar Energy Conversion (J. Schmid, H. A. Ossenbrink, P. Helm, H. Ehmann, and E. D. Dunlop, eds.), European Commission, Ispra, 1998, p. 1272.

[8] T. J. Rinke, R. B. Bergmann, R. Brüggemann, and J. H. Werner, Solid State Phenom. 67-68 (1999),
229.

[9] T. J. Rinke, R. B. Bergmann, and J. H. Werner, Appl. Phys. A 68 (1999), 705.

[10] T. J. Rinke, R. B. Bergmann, and J. H. Werner, Mat. Res. Soc. Symp. Proc. (1999), in press.

[11] R. Brendel, Proc. 12th Europ. Photovoltaic and Solar Energy Conference (R. Hill, W. Palz, and P. Helm, eds.), Stephens, Bedford, 1994, p. 1339.

[12] D. A. Clugston and P. A. Basore, Proc. 26th IEEE Photovoltaic Specialists Conference, IEEE, Piscataway, 1997, p. 207. 


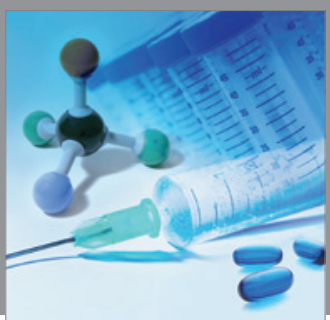

International Journal of

Medicinal Chemistry

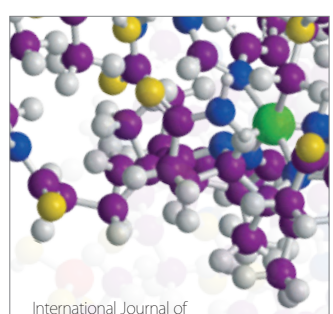

Carbohydrate Chemistry

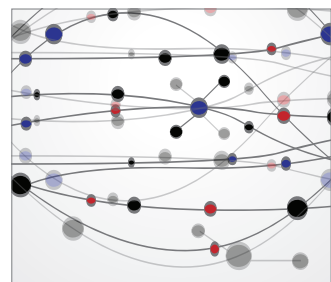

The Scientific World Journal
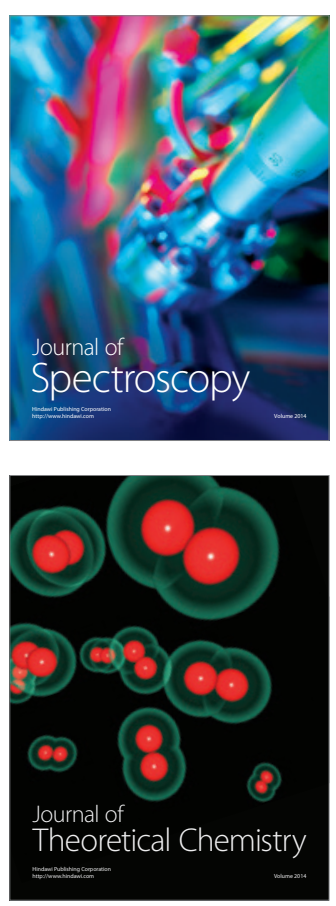
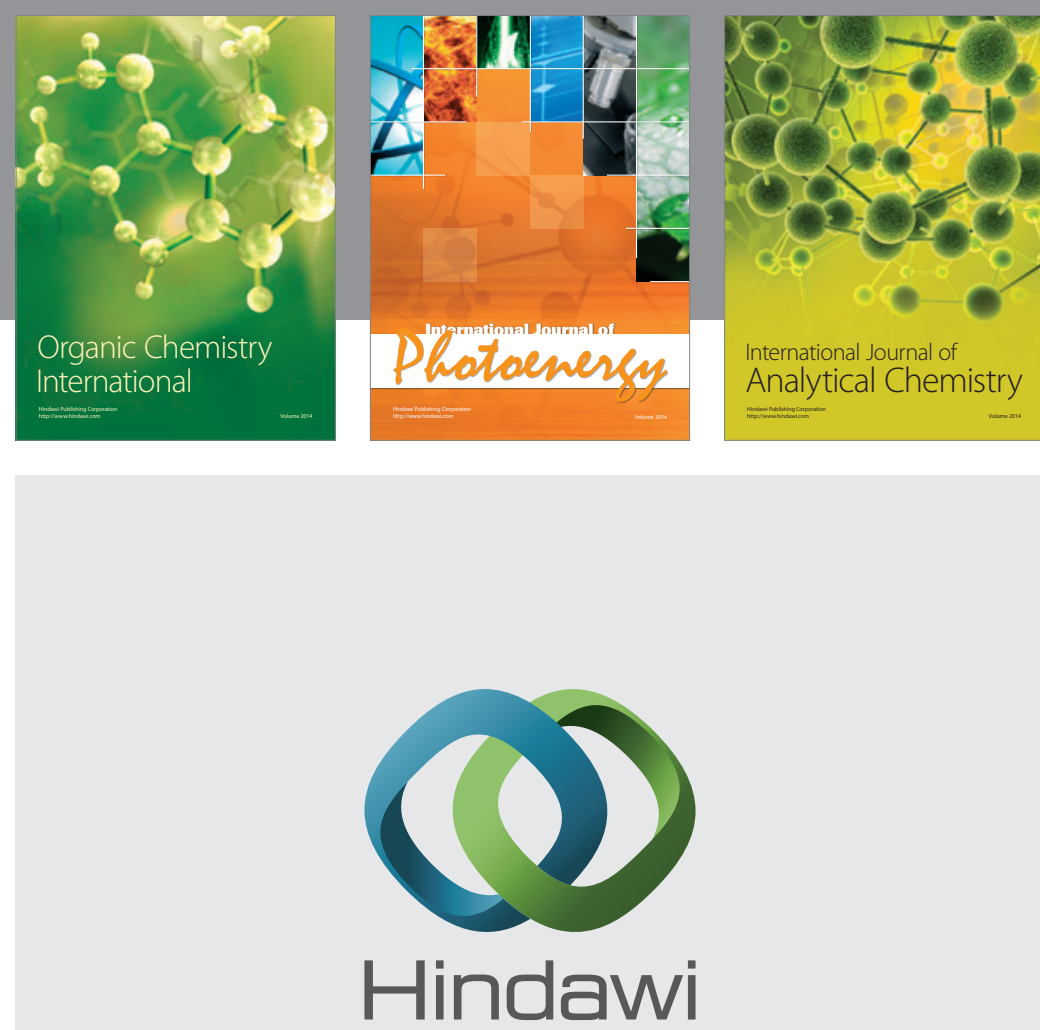

Submit your manuscripts at

http://www.hindawi.com
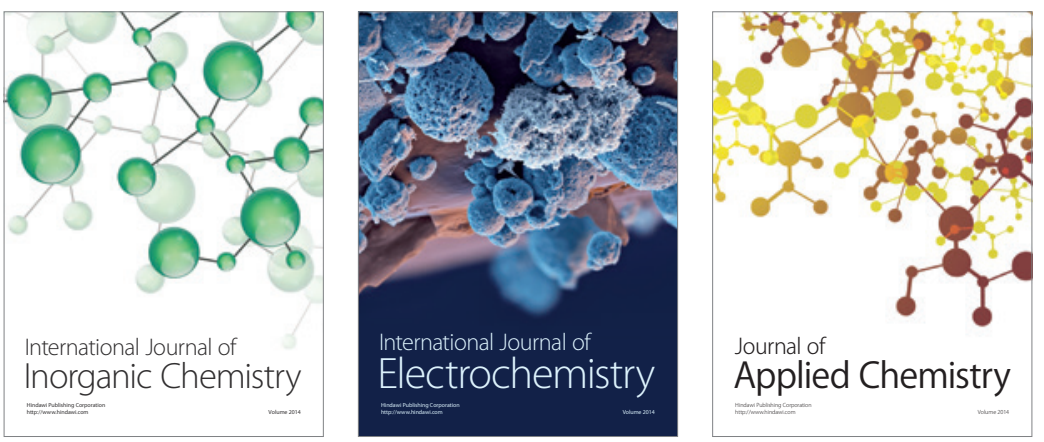

Journal of

Applied Chemistry
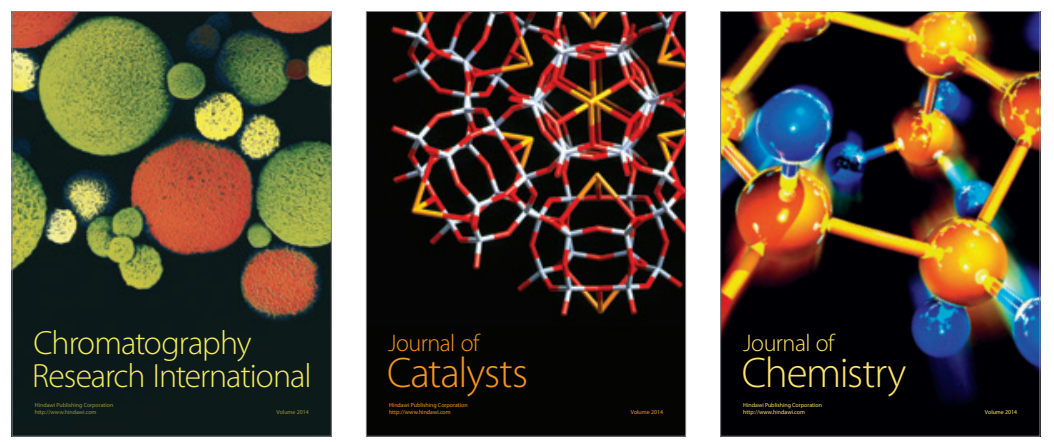
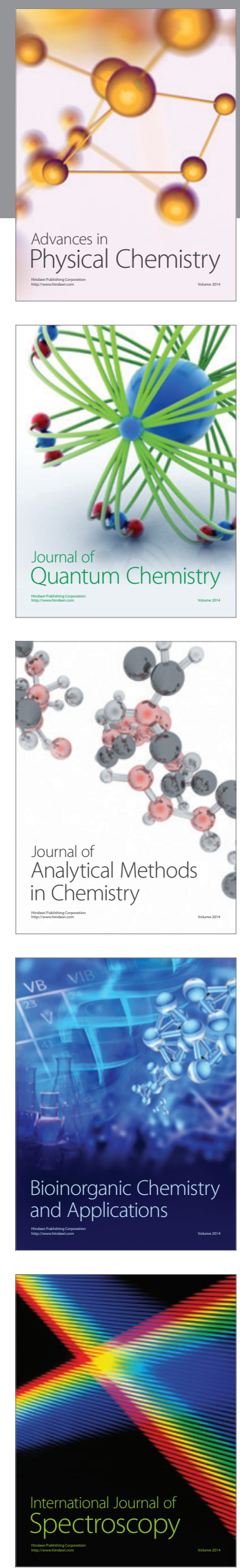